\title{
Use of durable left ventricular assist devices for high-risk patients: Korean experience before insurance coverage
}

\author{
Jun Ho Lee ${ }^{1}$, Ilkun Park ${ }^{2}$, Heemoon Lee ${ }^{3}$, Kiick Sung ${ }^{2}$, Young Tak Lee ${ }^{2}$, Darae Kim ${ }^{4}$ Jeong Hoon Yang \\ Jin-Oh Choi ${ }^{4}$, Eun-Seok Jeon ${ }^{4}$, Yang Hyun $\mathrm{Cho}^{2}$ \\ ${ }^{1}$ Department of Thoracic and Cardiovascular Surgery, Hanyang University Medical Center, Hanyang University School of Medicine, Seoul, Korea; \\ ${ }^{2}$ Department of Thoracic and Cardiovascular Surgery, Samsung Medical Center, Sungkyunkwan University School of Medicine, Seoul, Korea; \\ ${ }^{3}$ Department of Thoracic and Cardiovascular Surgery, Sejong General Hospital, Bucheon, Gyeonggi-do, Korea; ${ }^{4}$ Department of Cardiology, \\ Samsung Medical Center, Sungkyunkwan University School of Medicine, Seoul, Korea \\ Contributions: (I) Conception and design: JH Lee, YH Cho; (II) Administrative support: YH Cho; (III) Provision of study materials or patients: JH \\ Lee, I Park, H Lee, YT Lee; (IV) Collection and assembly of data: JH Lee, K Sung, JH Yang, YH Cho; (V) Data analysis and interpretation: JH Lee, \\ D Kim, JO Choi, ES Jeon; (VI) Manuscript writing: All authors; (VII) Final approval of the manuscript: All authors. \\ Correspondence to: Yang Hyun Cho, MD, PhD. Department of Thoracic and Cardiovascular Surgery, Samsung Medical Center, Sungkyunkwan \\ University School of Medicine, 81 Irwon-ro, Gangnam-gu, Seoul 06351, Korea. Email: mdcho95@gmail.com.
}

Background: Left ventricular assist devices (LVADs) were not covered by the Korean national insurance until September 2018, and they were implanted at the patient's own or a third party's expense. However, there have been no reports on using an LVAD without insurance coverage or manufacturer support.

Methods: We reviewed 23 patients who underwent durable LVAD implantation at our institution from August 2012 to September 2018. Patients with temporary LVADs using extracorporeal or paracorporeal circulation were excluded. The available devices were the HeartMate $\mathrm{II}^{\mathrm{TM}}$ (HMII) and HeartWare ${ }^{\mathrm{TM}}$ Ventricular Assist Device (HVAD). The primary outcome was 30-day mortality. The secondary outcomes were postoperative complications and late mortality.

Results: The mean age of the patients was $68.7 \pm 9.9$ years. The study sample comprised six female $(26.1 \%)$ and 17 male (73.9\%) patients. All patients had modifiable (bridge to candidacy) or unmodifiable absolute (destination therapy) contraindications for heart transplantation (HT). Among the patients in this study, 12 (52.2\%) had ischemic cardiomyopathy and 11 (47.8\%) had non-ischemic cardiomyopathy. Nine patients (39.1\%) had temporary mechanical circulatory support such as extracorporeal membrane oxygenation or a temporary LVAD in place preoperatively. The average duration of LVAD support was $618.6 \pm 563.2$ days (range, 59-2,285 days). There was no 30-day mortality. Four patients (17.4\%) underwent HT. Six patients (26.1\%) underwent re-exploration for postoperative bleeding, and one patient (4.3\%) had a disabling stroke after discharge. The estimated survival rates at 12 and 24 months were $89.2 \%$ and $68.8 \%$, respectively.

Conclusions: All patients who received LVADs before insurance coverage had contraindications for HT. The overall outcomes were comparable with those reported in the international registry.

Keywords: Left ventricular assist device (LVAD); heart transplantation; end-stage heart failure; destination therapy; bridge to transplant

Submitted Mar 22, 2020. Accepted for publication Jul 29, 2020.

doi: $10.21037 /$ jtd-20-1429

View this article at: http://dx.doi.org/10.21037/jtd-20-1429

(C) Journal of Thoracic Disease. All rights reserved. 


\section{Introduction}

Implantable left ventricular assist devices (LVADs) have become an established strategy for patients with end-stage heart failure to improve their functional capacity, quality of life, and survival rate (1-5). Therefore, LVADs are covered by insurance in several countries $(6,7)$. Because an LVAD is an expensive medical device, it is rarely implanted without insurance coverage or research funds. In South Korea, neither the national insurance nor LVAD manufacturers supported the cost of the device or covered the surgical fee until September 2018.

Our institution initiated a hospital-driven continuousflow LVAD trial (8). The trial aimed to garner the approval of the Korea Food and Drug Administration (KFDA) to use LVADs clinically. We continued to challenge the fact that LVAD implantation was not covered by national insurance, and we successfully obtained approval for national insurance coverage in September 2018.

Because LVAD implantations were performed at the patients' own expenses in most cases, it would be financially challenging for several patients in this extreme situation. We believe that the findings of this study will be meaningful in countries where LVADs are not yet covered by national insurance. We present the following article in accordance with the STROBE reporting checklist (available at http:// dx.doi.org/10.21037/jtd-20-1429).

\section{Methods}

\section{Introduction of continuous LVAD in South Korea}

Samsung Medical Center (SMC) is a tertiary referral center located in Seoul, South Korea. Although most Korean cardiologists and cardiac surgeons felt an increasing need for durable LVADs, no manufacturer or national insurance wanted to perform the clinical trials that were requested by the KFDA. In 2011, the LVAD trial of SMC was approved by the KFDA. After 2012, two kinds of continuous-flow devices, namely, the HeartMate IITM (HMII; Thoratec Corp., Pleasanton, CA, USA) ventricular assist system and HeartWareTM Ventricular Assist Device (HVAD; HeartWare International, Inc., Framingham, MA, USA), have been approved for either investigational or clinical purposes. Three consecutive LVAD implantations were performed with the support of our institution. Because of the favorable outcomes of the trial, the KFDA approved LVADs for clinical use in patients with end-stage heart failure. Until September 2018, all LVAD implantations in Korea were performed at either the patients' or a third party's expense such as a hospital or charity fund.

\section{Patient selection}

We reviewed 23 patients who underwent durable LVAD implantations at our institution from August 2012 to September 2018. Patients who had temporary LVADs using extracorporeal or paracorporeal circulation were excluded. At our institution, an LVAD was recommended only when the patient had a relative or absolute contraindication for heart transplantation (HT). Relatively stable young patients on inotropes who are awaiting HT are not usually interested in receiving LVADs because of their high financial burden. Patients who had the most urgent need for HT including unstable ones on extracorporeal membrane oxygenation (ECMO) or ventilatory support had a mean wait time for HT of approximately 1-2 weeks. Therefore, they waited for a suitable donor while on ECMO, intra-aortic balloon pump, or ventilator support. However, after 2015, the wait time for HT has rapidly increased, and Korean cardiologists began recommending LVAD implantation for some patients (Figure 1). At our institution, we usually recommend an LVAD as a bridge to candidacy (BTC) when there is a contraindication for HT such as high pulmonary vascular resistance (transpulmonary gradient $>15 \mathrm{mmHg}$ or pulmonary vascular resistance $\geq 6$ wood units), relapsing pneumonia, severe malnutrition or deconditioning, recent malignancy, recent alcoholism, and moderate chronic pulmonary disease. In patients older than 70 years, we usually discourage HT and recommend LVAD implantation as a as destination therapy (DT). We followed the previously published guidelines on LVAD (9-11). We were somewhat generous in following the contraindications because there was no strict control from insurance or the medical society. The final decision for LVAD placement was made by a multidisciplinary heart failure team. Although we suggested LVAD implantation to patients, some refused because of the financial burden or fear of surgery.

\section{Surgical technique and postoperative management}

In 20 patients, full median sternotomy was performed, and cardiopulmonary bypass $(\mathrm{CPB})$ was supported by a routine method with ascending aorta cannulation and bicaval venous drainage. In three patients, upper partial sternotomy and left anterior thoracotomy were performed. One patient had severe complications related to ECMO or 


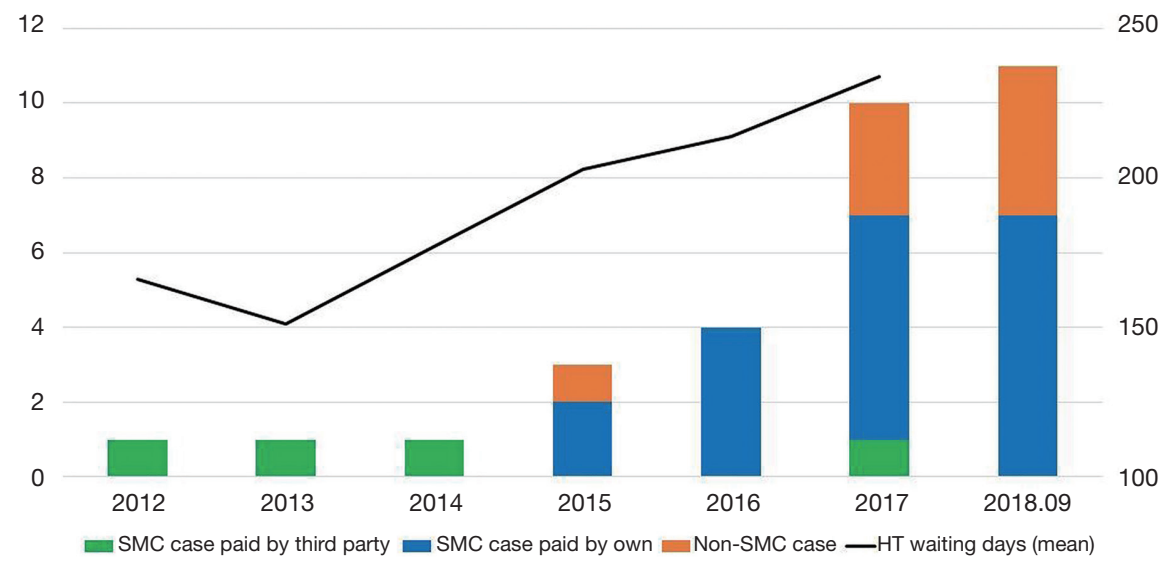

Figure 1 Left ventricular assist device (LVAD) implantation and waiting period for heart transplantation in Korea. After a waiting period of more than 200 days, the number of LVAD cases increased. SMC, Samsung Medical Center; HT, heart transplantation.
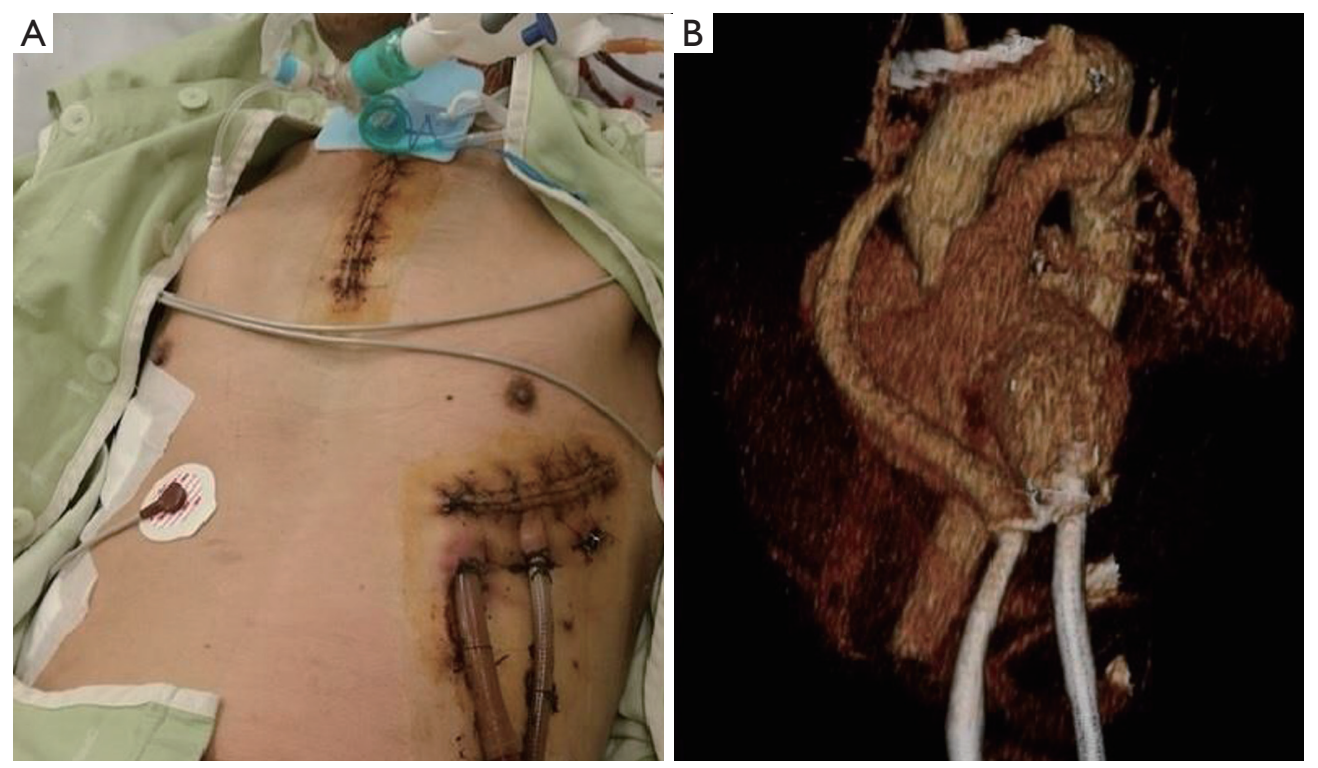

Figure 2 One patient with an extracorporeal left ventricular assist device (LVAD) placed through upper partial sternotomy and minithoracotomy. (A) Incision and positions of the cannulas in extracorporeal LVAD; (B) three-dimensional computed tomography reconstruction. A durable LVAD was implanted through the left thoracotomy without reopening the upper sternotomy.

critical care including limb ischemia, colonization by multi drug-resistant bacteria, and ventilator dependency from another hospital. We performed an extracorporeal LVAD implantation through partial sternotomy and thoracotomy (Figure 2). After ensuring the positive effects of the LVAD, the extracorporeal LVAD was converted to an HVAD through redo-thoracotomy (Video 1). In 2 of the 6 patients who received HMII, we placed the blood pump in the pericardial space. In the other four patients with the HMII, the pump was placed in the large deep lateral preperitoneal pocket (10). For drive-line tunneling, we generally used the double-tunnel technique (12).

In the immediate postoperative period, after confirming that there was no evidence of postoperative bleeding, intravenous heparin was started, and an activated partial thromboplastin time ranging from 60 to 70 seconds was achieved. We performed antiplatelet therapy with a daily dose of $100 \mathrm{mg}$ of acetylsalicylic acid. The dose of warfarin 
Table 1 Baseline characteristics and perioperative risk factors of all patients

\begin{tabular}{|c|c|c|c|}
\hline Variables & DT $(n=15)$ & BTC $(n=8)$ & Total $(n=23)$ \\
\hline \multicolumn{4}{|l|}{ Age (years) } \\
\hline Median [range] & 75 [66-81] & $62[47-67]$ & $71[47-81]$ \\
\hline$>65$ years & 15 & 1 & $16(69.6 \%)$ \\
\hline \multicolumn{4}{|l|}{ Gender } \\
\hline Male & 11 & 6 & $17(73.9 \%)$ \\
\hline Female & 4 & 2 & $6(26.1 \%)$ \\
\hline \multicolumn{4}{|c|}{ Preoperative diagnosis } \\
\hline ICMP & 7 & 5 & $12(52.2 \%)$ \\
\hline Non-ICMP & 8 & 3 & $11(47.8 \%)$ \\
\hline \multicolumn{4}{|l|}{ INTERMACS profile } \\
\hline 1 & 2 & 1 & $3(13.0 \%)$ \\
\hline 2 & 6 & 4 & $10(43.5 \%)$ \\
\hline 3 & 7 & 3 & $10(43.5 \%)$ \\
\hline \multicolumn{4}{|l|}{ Reason for LVAD } \\
\hline Old age & 12 & 0 & $12(52.2 \%)$ \\
\hline Infection & 2 & 4 & $6(26.1 \%)$ \\
\hline COPD & 1 & 0 & $1(4.3 \%)$ \\
\hline CKD & 0 & 1 & $1(4.3 \%)$ \\
\hline Malignancy & 0 & 1 & $1(4.3 \%)$ \\
\hline Substance abuse & 0 & 1 & $1(4.3 \%)$ \\
\hline Foreigner & 0 & 1 & $1(4.3 \%)$ \\
\hline Temporary MCS & $4(26.7 \%)$ & $5(62.5 \%)$ & $9(39.1 \%)$ \\
\hline CPR & 6 & 3 & $9(39.1 \%)$ \\
\hline HTN & 10 & 4 & $14(60.9 \%)$ \\
\hline DM & 7 & 4 & $11(47.8 \%)$ \\
\hline Dialysis & 3 & 3 & $6(26.1 \%)$ \\
\hline
\end{tabular}

DT, destination therapy; BTC, bridge to candidacy; ICMP, ischemic cardiomyopathy; INTERMACS, Interagency Registry for Mechanically Assisted Circulatory Support; LVAD, left ventricular assist device; COPD, chronic obstructive pulmonary disease; CKD, chronic kidney disease; MCS, mechanical circulatory support; CPR, cardiopulmonary resuscitation; HTN, hypertension; DM, diabetes mellitus. was adjusted to maintain an international normalized ratio of 2.0-2.5.

\section{Definitions and follow-up}

The patients who underwent LVAD implantation as a DT due to old age or other causes of unmodifiable absolute contraindications for HT were assigned to the DT group. Consequently, those who had modifiable contraindications for HT and underwent LVAD implantation as a BTC were assigned to the BTC group.

The primary outcome of this study was 30-day mortality. The secondary outcomes were postoperative complications and late mortality. Postoperative complications included postoperative right ventricular assist device (RVAD) implantation, bleeding, disabling stroke (with "disabling" defined as a modified Rankin scale score of $>4$; scores range from 0 to 6 , with higher scores indicating more severe disability) $(9,11,13)$, readmission for the treatment of right heart failure, brain hemorrhage, bacteremia, aspiration pneumonia, device malfunction, driveline infection and thrombus in the device. The median follow-up period was 15 months.

Baseline parameters for clinical data were based on medical records and databases. Follow-up clinical data were acquired through medical record review and telephone interviews. To complete the follow-up data, including deaths, information was collected from the National Registry of Births and Deaths using a unique personal identification number. The study was conducted in accordance with the Declaration of Helsinki (as revised in 2013). The study was approved by institutional review board of SMC (IRB No.: SMC 2019-02-092), and the requirement for patient consent was waived.

\section{Results}

\section{Baseline characteristics}

The overall baseline characteristics and perioperative risk factors for the patients are summarized in Table 1. A total of 23 patients were included in this study. The mean age of 
Table 2 Operative outcomes

\begin{tabular}{|c|c|c|c|}
\hline Outcomes & DT $(n=15)$ & BTC $(n=8)$ & Total $(n=23)$ \\
\hline Postoperative ICU stay (median, days) & 10 & 26.5 & 13 \\
\hline Postoperative RVAD & 1 & 3 & $4(17.4 \%)$ \\
\hline 30-day mortality & 0 & 0 & $0(0.0 \%)$ \\
\hline Duration of LVAD (days) (mean \pm standard deviation) & $699.9 \pm 639.2$ & $466.3 \pm 373.2$ & $618.6 \pm 563.2$ \\
\hline Duration of LVAD (days), median [range] & $536[130-2,285]$ & $320.5[59-1,256]$ & $472[59-2,285]$ \\
\hline HeartMate $I^{\mathrm{TM}}$ & 5 & 1 & $6(26.1 \%)$ \\
\hline HVAD & 10 & 7 & $17(73.9 \%)$ \\
\hline
\end{tabular}

DT, destination therapy; BTC, bridge to candidacy; ICU, intensive care unit; RVAD, right ventricular assist device; LVAD, left ventricular assist device; HVAD, HeartWare ${ }^{\mathrm{TM}}$ Ventricular Assist Device.

the patients was $68.7 \pm 9.9$ years, and the median age was 71 years. The study sample comprised 6 female $(26.1 \%)$ and 17 male $(73.9 \%)$ patients. The devices we used were 8 the HMII $(\mathrm{n}=6,26.1 \%)$ or HVAD $(\mathrm{n}=17,73.9 \%)$. Nineteen patients $(82.6 \%)$ paid the device and surgical fees at their own expense. Based on the Interagency Registry for Mechanically Assisted Circulatory Support (INTERMACS), all patients were classified between level 1 and level 3 .

There were 15 patients $(65.2 \%)$ in the DT group and 8 patients $(34.8 \%)$ in the BTC group. Five patients $(62.5 \%)$ in the BTC group had preoperative temporary mechanical circulatory support (MCS) such as ECMO or extracorporeal LVAD. In the DT group, 6 patients (40.0\%) had a history of cardiopulmonary resuscitation (CPR), while in the BTC group, $3(37.5 \%)$ had a history of CPR. Three patients (20.0\% and $37.5 \%$, respectively) in each the DT and BTC groups had a history of dialysis.

Patient profiles are summarized in Table S1. A foreign patient needed LVAD implantation because they could not be placed on the waiting list for HT. Although we recommended HT for ischemic cardiomyopathy (ICMP) and inotrope dependency, we could not perform HT because of her foreign nationality.

Seven patients $(30.4 \%)$ underwent LVAD implantation via redo-sternotomy. The mean CPB time was 106.0 \pm 49.0 minutes, and the median time was 93.0 minutes. Concomitant procedures were primary closure of an atrial septal defect or patent foramen ovale in $4(17.4 \%)$ patients, tricuspid annuloplasty in $3(13.0 \%)$ patients, intracardiac thrombus removal in $2(8.7 \%)$ patients, aortic valve replacement in 2 $(8.7 \%)$ patients, aortic valve closure in $2(8.7 \%)$ patients, and hemiarch replacement in $1(4.3 \%)$ patient.

\section{Clinical outcomes}

The overall perioperative outcomes for all patients are summarized in Table 2. Four patients (17.4\%) had severe right ventricular dysfunction that required RVAD support. Three patients $(13.0 \%)$ had a temporary RVAD in the operating room. One patient (4.3\%) had an RVAD system in the intensive care unit (ICU). The median duration of RVAD support was 8.5 days. All aforementioned patients were successfully weaned from the RVAD support.

There was no 30-day mortality, and no patients died during hospitalization after LVAD implantation. Four patients died during the follow-up period. A 73-year-old man in the DT group underwent unplanned HT (after 23 months) but died 15 days after HT. A 48-year-old woman in the BTC group died 10 months after LVAD implantation due to progression of malignancy. An 81-year-old man in the DT group died 17 months after LVAD implantation due to pump power loss for unknown reasons. A 47-year-old man in the BTC group died 8 months after surgery due to severe cerebral dysfunction from severe alcoholism. The estimated survival rates at 12 and 24 months were $89.2 \%$ and $68.8 \%$, respectively (Figure $3 A$ ). Seventyfive-point-four percent had freedom from death or unplanned HT at 12 months and $48.5 \%$ at 24 months (Figure 3B). The average duration of LVAD support was 618.6 \pm 563.2 days (range, 59-2,285; median, 472 days). Postoperative complications are summarized in Table 3. Regarding hospital complications, there were 6 cases 

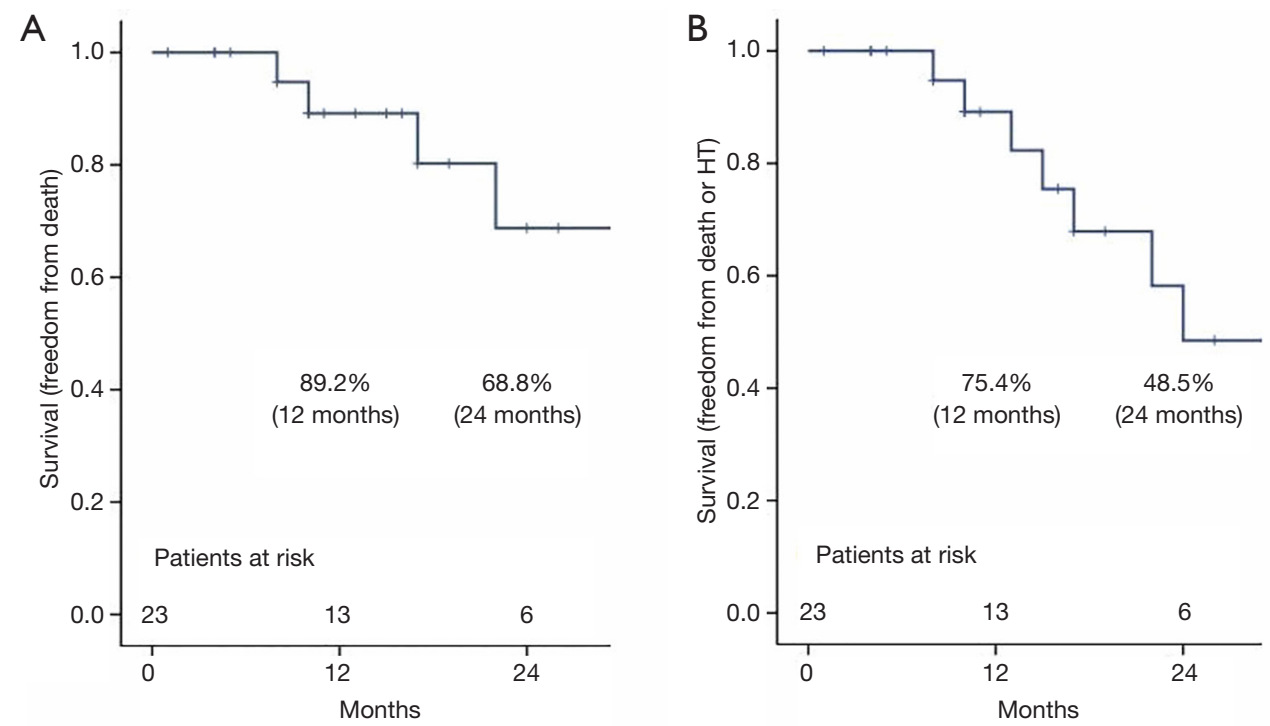

Figure 3 Kaplan-Meier estimates of all patients. (A) Freedom from death; (B) freedom from death or heart transplantation.

Table 3 Complications after left ventricular assist device implantation

\begin{tabular}{lccc}
\hline Complications & In-hospital & After discharge & \\
\hline Bleeding & 6 & 0 & 6 \\
Re-exploration for postoperative bleeding & 6 & 1 & 11 \\
Non-surgical (gastrointestinal) & 0 & 1 & 1 \\
Disabling stroke & 0 & 1 & 3 \\
Readmission for right heart failure treatment & 2 & 1 & 3 \\
Bacteremia & 2 & 1 & 1 \\
Pneumonia & 0 & 1 & 1 \\
Driveline infection & 0 & 0 \\
Device infection & 0 & 0 \\
Device malfunction & 0 & 1 \\
Confirmed device thrombosis & & 1 \\
\hline
\end{tabular}

(26.1\%) of bleeding control, 2 cases $(8.7 \%)$ of stroke, and 6 cases $(26.1 \%)$ of gastrointestinal bleeding. Regarding late complications after hospital discharge, there were 4 cases $(17.4 \%)$ of stroke, 1 case $(4.3 \%)$ of driveline infection, and 1 case $(4.3 \%)$ of device infection.

Four patients (two in the DT group and two in the BTC group) who underwent HT. A 73-year-old man in the DT group had a device malfunction (inflow cannula obstruction) and underwent HT 23 months after LVAD implantation. A 69-year-old man in the DT group had a device infection and underwent HT 13 months after LVAD implantation. A 49-year-old man and a 62-year-old man in the BTC group underwent HT due to unsatisfactory LVAD flow and heart failure. There were no cases of confirmed device thrombosis during the study period including outpatient follow-up.

\section{Discussion}

In this study, we demonstrated our early experiences with durable LVAD implantation in our institution. Most of 
the LVAD implantations in Korea were performed by our group during the study period (Figure 1). Although studies from other Asian countries have been reported, this study is the first collective review including only DT or bridge to transplant (BTT) patients $(4,5)$. Because all LVAD implantations were performed before the Korean national insurance coverage was approved, patient profiles were relatively unique. There was no frank BTT case. All patients had clear contraindications for HT. In this study, LVADs were used as a DT in $65.2 \%$ of the patients. Although no definite BTT patients were present, the remaining $34.8 \%$ of the patients had modifiable contraindications for HT, such as recent cancer diagnosis, substance abuse, very advanced age, and active infection.

In this study, 12 patients $(52.2 \%)$ who underwent durable LVAD implantation were over 70 years old. Additionally, 2 patients (8.7\%) were over 80 years old. Elderly patients who were inappropriate candidates for HT ventured on LVADs in spite of their high cost and risk. It seems that LVAD implantation as a DT is gradually becoming popular worldwide, and the rate of LVAD implantation in elderly patients has increased, as reported in a previous publication (14).

In South Korea, we are also experiencing a shortage of organ donors relative to the number of patients waiting for transplantation. Therefore, the number of unstable patients is increasing. According to the Korea Centers for Disease Control and Prevention, the 1-year survival rate in patients with the most urgent need for HT was $72.8 \%$, which is significantly lower than that of the entire cohort (86.0\%) (15).

In the BTC group, eight patients had modifiable contraindications for HT, and 5 patients $(62.5 \%)$ had ECMO before LVAD implantation. Moreover, in the DT group, 4 patients (26.7\%) had temporary MCS before LVAD implantation. In published papers, BTT with ECMO was associated with a poorer post-HT survival rate, which seems to be similar to the results of this study $(16,17)$. Now that medical insurance covers LVAD implantation, the use of ECMO for BTT should be reduced and it can be expected that there will be a more efficient use of transplanted organs.

In our data, 6 patients $(26.1 \%)$ had preoperative infections. Most of their infections were related to their general condition and critical care, such as the use of ventilators, catheters, and ECMO. LVADs may be associated with infectious complications and are contraindicated in some patients receiving immunosuppressive therapy. Therefore, our patients had various non-LVAD-related and
LVAD-related infectious complications such as bacteremia in 3 patients (13.0\%), pneumonia in three patients $(13.0 \%)$, driveline infection in 1 patient $(4.3 \%)$, and device infection in 1 patient $(4.3 \%)$. One patient had a delayed device infection, which was treated with debridement and vacuumassisted closure. The infection recurred 2 months after closure, and we performed urgent HT and device removal. The patient who had a driveline infection was successfully managed with exit-site switching and vacuum-assisted closure. Gordon and colleagues reported that ventricular assist device (VAD)-related infections had an incidence of 0.10 per 100 person-days and increased 1-year mortality (18). The two patients with LVAD-related infections were successfully managed.

Finally, in all patients, the estimated survival rates at 12 and 24 months were $89.2 \%$ and $68.8 \%$, respectively. The eighth annual INTERMACS report demonstrated that the overall survival rates for patients who underwent LVAD implantation (with or without RVAD) were $81 \%$ at 12 months and $70 \%$ at 24 months (19). Furthermore, the first annual International Society for Heart and Lung Transplantation Registry for Mechanically Assisted Circulatory Support (IMACS) report demonstrated that the overall survival rates for patients receiving durable LVADs were $80 \%$ at 12 months and $75 \%$ at 18 months (20). Considering the patient group with a large portion of DT and low INTERMACS levels, our results are acceptable compared with other international registries $(19,20)$.

This study has several recognized limitations and our results need to be interpreted prudently. First, our database included a small number of patients, and it has limited statistical power with the lack of data. Because it reflects the early experience of our institution, the number of samples should be insufficient for further evaluation and could increase the risk of bias. Additional clinical research in South Korea should be conducted to investigate whether LVAD implantation with medical insurance coverage can contribute to the survival and quality of life of patients with end-stage heart failure as a BTT and DT. A large randomized and prospective multicenter study with longterm close follow-up is warranted. Second, the follow-up period might not have been long enough to evaluate the long-term clinical outcomes. Because our investigation was retrospective in nature and the number of patients who underwent LVAD implantation has increased exponentially since 2017 , it was difficult to assess the long-term clinical results of all patients.

In conclusion, all patients who received LVADs before 
insurance coverage had contraindications for HT. The overall outcomes were comparable with those reported in the international registry.

\section{Acknowledgments}

The authors would like to thank Sujin Kim from the Department of Thoracic and Cardiovascular Surgery at SMC for collecting the necessary data and providing statistical support.

Funding: None.

\section{Footnote}

Reporting Checklist: The authors have completed the STROBE reporting checklist. Available at http://dx.doi. org/10.21037/jtd-20-1429

Data Sharing Statement: Available at http://dx.doi. org/10.21037/jtd-20-1429

Conflicts of Interest: All authors have completed the ICMJE uniform disclosure form (available at http://dx.doi. org/10.21037/jtd-20-1429). The authors have no conflicts of interest to declare.

Ethical Statement: The authors are accountable for all aspects of the work in ensuring that questions related to the accuracy or integrity of any part of the work are appropriately investigated and resolved. The study was conducted in accordance with the Declaration of Helsinki (as revised in 2013). The study was approved by institutional review board of SMC (IRB No.: SMC 2019-02-092), and the requirement for patient consent was waived.

Open Access Statement: This is an Open Access article distributed in accordance with the Creative Commons Attribution-NonCommercial-NoDerivs 4.0 International License (CC BY-NC-ND 4.0), which permits the noncommercial replication and distribution of the article with the strict proviso that no changes or edits are made and the original work is properly cited (including links to both the formal publication through the relevant DOI and the license). See: https://creativecommons.org/licenses/by-nc-nd/4.0/.

\section{References}

1. Slaughter MS, Rogers JG, Milano CA, et al. Advanced heart failure treated with continuous-flow left ventricular assist device. N Engl J Med 2009;361:2241-51.

2. Magruder JT, Grimm JC, Crawford TC, et al. Survival After Orthotopic Heart Transplantation in Patients Undergoing Bridge to Transplantation with the HeartWare HVAD Versus the Heartmate II. Ann Thorac Surg 2017;103:1505-11.

3. Pagani FD, Lynch W, Swaniker F, et al. Extracorporeal life support to left ventricular assist device bridge to heart transplant: A strategy to optimize survival and resource utilization. Circulation 1999;100:II206-10.

4. Nishi H, Toda K, Miyagawa S, et al. Initial experience in Japan with HeartWare ventricular assist system. J Artif Organs 2014;17:149-56.

5. Lim CP, Sivathasan C, Tan TE, et al. Use of left ventricular assist device (HeartMate II): a Singapore experience. Artif Organs 2014;38:543-8.

6. Pagani FD, Miller LW, Russell SD, et al. Extended mechanical circulatory support with a continuous-flow rotary left ventricular assist device. J Am Coll Cardiol 2009;54:312-21.

7. Lund LH, Khush KK, Cherikh WS, et al. The Registry of the International Society for Heart and Lung Transplantation: Thirty-fourth Adult Heart Transplantation Report-2017; Focus Theme: Allograft ischemic time. J Heart Lung Transplant 2017;36:1037-46.

8. Lee GY, Park SJ, Kim S, et al. The successful implantation of continuous-flow left ventricular assist device as a destination therapy in Korea: echocardiographic assessment. J Korean Med Sci 2014;29:137-40.

9. Rogers JG, Pagani FD, Tatooles AJ, et al. Intrapericardial Left Ventricular Assist Device for Advanced Heart Failure. N Engl J Med 2017;376:451-60.

10. John R, Kamdar F, Liao K, et al. Improved survival and decreasing incidence of adverse events with the HeartMate II left ventricular assist device as bridge-to-transplant therapy. Ann Thorac Surg 2008;86:1227-34; discussion 1234-5.

11. Mehra MR, Naka Y, Uriel N, et al. A Fully Magnetically Levitated Circulatory Pump for Advanced Heart Failure. N Engl J Med 2017;376:440-50.

12. Schibilsky D, Benk C, Haller C, et al. Double tunnel technique for the LVAD driveline: improved management regarding driveline infections. J Artif Organs 2012;15:44-8.

13. Mehra MR, Goldstein DJ, Uriel N, et al. Two-Year Outcomes with a Magnetically Levitated Cardiac Pump in Heart Failure. N Engl J Med 2018;378:1386-95. 
14. Atluri P, Goldstone AB, Kobrin DM, et al. Ventricular assist device implant in the elderly is associated with increased, but respectable risk: a multi-institutional study. Ann Thorac Surg 2013;96:141-7.

15. Korea Centers for Disease Control and Prevention. Korean Network for Organ Sharing. [cited 2019 Mar 1]. Available online: https://www.konos.go.kr/konosis/

16. Fukuhara S, Takeda K, Kurlansky PA, et al. Extracorporeal membrane oxygenation as a direct bridge to heart transplantation in adults. J Thorac Cardiovasc Surg 2018;155:1607-1618.e6.

17. Zalawadiya S, Fudim M, Bhat G, et al. Extracorporeal membrane oxygenation support and post-heart transplant outcomes among United States adults. J Heart Lung

Cite this article as: Lee JH, Park I, Lee H, Sung K, Lee YT, Kim D, Yang JH, Choi JO, Jeon ES, Cho YH. Use of durable left ventricular assist devices for high-risk patients: Korean experience before insurance coverage. J Thorac Dis 2020;12(12):7236-7244. doi: 10.21037/jtd-20-1429
Transplant 2017;36:77-81.

18. Gordon RJ, Weinberg AD, Pagani FD, et al. Prospective, multicenter study of ventricular assist device infections. Circulation 2013;127:691-702.

19. Kirklin JK, Pagani FD, Kormos RL, et al. Eighth annual INTERMACS report: Special focus on framing the impact of adverse events. J Heart Lung Transplant 2017;36:1080-6.

20. Kirklin JK, Cantor R, Mohacsi P, et al. First Annual IMACS Report: A global International Society for Heart and Lung Transplantation Registry for Mechanical Circulatory Support. J Heart Lung Transplant 2016;35:407-12. 


\section{Supplementary}

Table S1 Profile of all patients

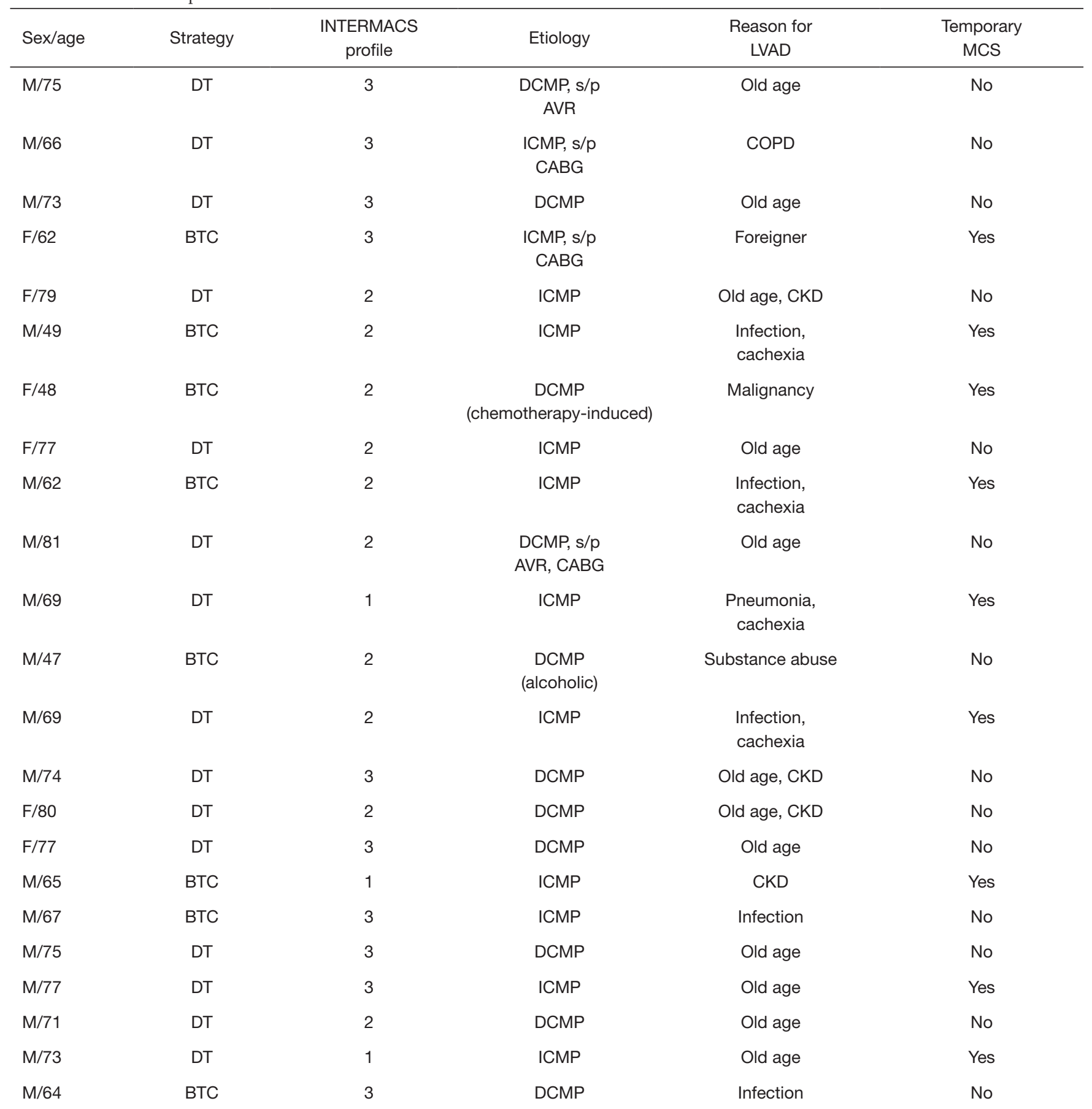

INTERMACS, Interagency Registry for Mechanically Assisted Circulatory Support; LVAD, left ventricular assist device; MCS, mechanical circulatory support; DT, destination therapy; DCMP, dilated cardiomyopathy; AVR, aortic valve replacement; ICMP, ischemic cardiomyopathy; CABG, coronary artery bypass grafting; COPD, chronic obstructive pulmonary disease; BTC, bridge to candidacy; CKD, chronic kidney disease; $s / p$, status post. 